\title{
Key genes in youth colorectal cancer based on data mining and verification by reverse transcription-quantitative PCR
}

\author{
JIANXIN CHEN ${ }^{1,2}$, MIN LIN $^{3}$, YAN CHE $^{3,4}$, JIAN GUO $^{1,2}$ and WEI LIN ${ }^{1,2}$ \\ ${ }^{1}$ The First Department of Gastrointestinal Surgery; ${ }^{2}$ Gastrointestinal Surgery Research \\ Institute, The Affiliated Hospital of Putian University; ${ }^{3}$ College of Information Engineering, \\ Putian University; ${ }^{4}$ Engineering Research Center of Big Data Application in Private Health Medicine, \\ Fujian Province University, Putian, Fujian 351100, P.R. China
}

Received July 10, 2020; Accepted December 7, 2020

DOI: $10.3892 / \mathrm{ol} .2021 .12455$

\begin{abstract}
In recent years, among all patients with colorectal cancer, the proportion of young patients has been gradually increasing. However, the molecular mechanisms involved in colorectal cancer in the young are largely unknown. In the present study the Gene Expression Omnibus (GEO) and The Cancer Genome Atlas datasets were integrated to elucidate the key gene biomarkers in these patients. The GSE41657 and GSE41258 datasets were downloaded from the GEO database. By screening for differentially expressed genes, Gene Ontology analysis, Kyoto Encyclopedia of Genes and Genomes pathway analysis, protein-protein interaction analysis, hub gene screening and survival analysis, two key genes, CXCL8 and VEGFA, which were enriched in cancer pathways, were obtained. Reverse transcription-quantitative (RT-q)PCR was performed to verify the outcome obtained by bioinformatics analysis. In conclusion, the present study identified two key genes using bioinformatics analysis and RT-qPCR validation. These results indicated that the candidate genes may be involved in the progression of colorectal cancer in young people, and these two genes may act as ideal prognostic indicators or therapeutic targets for colorectal cancer in the youth.
\end{abstract}

\section{Introduction}

Colorectal cancer is a common malignant tumor of the digestive tract. Present statistics show that colorectal cancer

Correspondence to: Professor Wei Lin, The First Department of Gastrointestinal Surgery, The Affiliated Hospital of Putian University, 999 Dongzhen Road, Putian, Fujian 351100, P.R. China E-mail: linwbj@outlook.com

Abbreviations: DEGs, differentially expressed genes; DAVID, Database for Annotation, Visualization and Integration Discovery; GEO, Gene Expression Omnibus; GO, Gene Ontology; KEGG, Kyoto Encyclopedia of Genes and Genomes; FC, fold change; TCGA, The Cancer Genome Atlas; HPA, Human Protein Atlas

Key words: colorectal cancer, GEO, bioinformatics analysis, DEGs, youth ranks third in incidence and forth in mortality rates among malignant tumors worldwide (1). In recent years, the age of onset of colorectal cancer has decreased. A survey showed that between 2000 and 2013, the incidence of colorectal cancer among Americans aged $<50$ increased by $22 \%$ (2).

Although an increasing number of measures for cancer prevention and treatment are being implemented, the incidence and mortality of colorectal cancer remain high (3). The development of precision medicine in recent years is expected to revolutionize the diagnosis and treatment of colorectal cancer (4). Identifying molecular markers corresponding to tumors and achieving individualized treatment to maximize efficacy and minimize side effects is an important direction for future clinical tumor treatment $(5,6)$. Colorectal cancer is caused by a variety of factors, such as a series of genetic events and gene mutations, and generally involves the activation of oncogenes and inactivation of tumor suppressor genes. Youth colorectal cancer has unique molecular characteristics. Mork et al (7) found that the proportion of youth colorectal cancer secondary to familial adenomatous polyposis with an APC gene mutation was significantly higher compared with colorectal cancer in the elderly. Compared with elderly colorectal cancer, youth colorectal cancer has unique molecular biological characteristics, including a high proportion of microsatellite high instability, a high proportion of mismatch repair gene defects and relatively lower mutation rates of the BRAF and KRAS genes in the EGFR pathway (8-10). However, the underlying molecular biological mechanism of young colorectal cancer is still not completely clear, rendering it necessary to explore specific molecular markers for colorectal cancer in young people. In the present study, Gene Expression Omnibus (GEO), The Cancer Genome Atlas (TCGA) database data mining and reverse transcription-quantitative (RT-q) PCR were used to explore key molecular markers for youth colorectal cancer.

\section{Materials and methods}

Microarray data. The GSE41657 (analyzed on Agilent-014850 Whole Human Genome Microarray 444K G4112F platform; conducted by Peking Union Medical College and Chinese Academy of Medical Sciences in China) and GSE41258 
(analyzed on Affymetrix Human Genome U133A Array platform; conducted by Weizmann Institute of Science in Israel) gene expression profiles in human colorectal cancer were downloaded from the GEO database of the National Center for Biotechnology Information (www.ncbi.nlm.nih.gov/geo). The patients selected for the cancer and normal groups were all aged $<50$ years.

Data preprocessing and screening strategy. Firstly, GEO2R was used to identify the differentially expressed genes (DEGs) between youth colorectal cancer and normal tissues. GEO2R (www.ncbi.nlm.nih.gov/geo/geo2r/) is a convenient web tool for DEG screening by comparing two groups of samples in a GEO dataset (11). An adjusted $\mathrm{P}<0.05$ and $\log _{2}$ fold change $(\mathrm{FC}) \mid \geq 1$ were set as the cut-off criteria.

Secondly, these DEGs were classified according to Gene Ontology (GO; http://geneontology.org/) and Kyoto Encyclopedia of Genes and Genomes (KEGG; www.genome. jp/kegg) pathways using Database for Annotation, Visualization and Integration Discovery (DAVID; https://david.ncifcrf.gov/) software. Based on the enriched GO terms and significant KEGG pathways, the disease-associated pathways and genes were screened.

Thirdly, DEGs were imported into STRING software (version 3.6.0; https://string-db.org/) to produce protein-protein interaction networks, and then Cytoscape software (version 3.6.0; https://cytoscape.org/) was used to construct a visible network diagram. In this network, cytoHubba plugin was used to screen the top 10 hub genes using degree algorithm. Combined with the analysis of signaling pathways, disease-associated genes were screened out.

TCGA, human protein atlas (HPA) validation and survival analysis. TCGA (http://cancergenome.nih.gov/) dataset was used to validate the disease-associated genes screened by GEO datasets. TCGA dataset was analyzed using Gene Expression Profiling Interactive Analysis (http://gepia.cancer-pku.cn/), which is a commonly used interactive website that plots expression profiles of selected genes. A total of 362 colorectal cancer and 51 normal tissues from TCGA database were selected for analysis. Survival analysis was further performed on these genes, based on gene expression levels, as previously described (12). Key genes were obtained by overall survival (OS) and disease-free survival (DFS) analysis, and the association between the expression of these genes and tumor staging was explored. Patients with high expression levels (50\%) of key genes were assigned to high expression group. The expression levels of key genes were also validated using the HPA database (http://www.proteinatlas.org/) (13).

$R T$-qPCR validation. Six pairs of youth colorectal cancer and adjacent normal tissues from patients aged $<50$ years (age range, 35-48; 3 men and 3 women) were collected from the First Department of Gastrointestinal Surgery of the Affiliated Hospital of Putian University (Putian, China). All colorectal cancer and normal tissues were stored in a liquid nitrogen tank within $30 \mathrm{~min}$ from their removal from the patient. For RNA extraction, the sample was ground into pieces in a mortar filled with liquid nitrogen. Total RNA was then extracted using RNAiso Plus (Takara Bio, Inc.) and reverse transcribed into
Table I. 10 hub genes ranked by degree.

\begin{tabular}{llcc}
\hline Gene name & Category & Score & Rank \\
\hline VEGFA & Upregulated & 65 & 1 \\
CXCL8 & Upregulated & 53 & 2 \\
MYC & Upregulated & 53 & 3 \\
CD44 & Upregulated & 43 & 4 \\
CXCL12 & Downregulated & 39 & 5 \\
CCND1 & Upregulated & 39 & 6 \\
IGF1 & Downregulated & 38 & 7 \\
CXCL1 & Upregulated & 32 & 8 \\
KIT & Downregulated & 30 & 9 \\
SOX9 & Upregulated & 29 & 10 \\
\hline
\end{tabular}

cDNA using PrimeScript ${ }^{\mathrm{TM}}$ RT reagent kit with gDNA Eraser (Takara Bio, Inc.), according to the manufacturer's instructions. RT-qPCR was performed using the SYBR Premix Ex Taq $^{\mathrm{TM}}$ II (Takara Bio, Inc.), according to the manufacturer's instructions. The PCR primers were designed by the National Center for Biotechnology Information (www.ncbi.nlm.nih. gov/) and provided by SangonBiotech Co., Ltd. (Table SI). The gene GAPDH was selected as an internal reference, to compare gene expression in different samples. The RT-qPCR process included the following steps: Pre-denaturation at $95^{\circ} \mathrm{C}$ for $30 \mathrm{sec}$, followed by 40 cycles of denaturation at $95^{\circ} \mathrm{C}$ for $5 \mathrm{sec}$ and extension at $60^{\circ} \mathrm{C}$ for $35 \mathrm{sec}(7500$ Real Time PCR System; Thermo Fisher Scientific, Inc.). Each sample was tested in triplicate. Gene expression values in each sample were calculated using the $2^{-\triangle \Delta C q}$ method (14). The present study was approved by the Ethics Committee of the Affiliated Hospital of Putian University (approval no. 202016). Written informed consent was obtained from each patient for sample collection and analysis.

Statistical analysis. SPSS version 17.0 (SPSS Inc.) was used to analyze the data. Continuous variables are presented as the mean \pm standard deviation. One-way ANOVA with least significant difference post hoc test was used to analyze the data of more than two groups. Student's t-test was used for comparisons between two groups. A paired t-test was used when comparing the six pairs of youth colorectal cancer and adjacent normal tissues, and an unpaired t-test was used for other comparisons. If the variance was not equal between two groups, Mann-Whitney U test was used for statistical analysis. The statistical significance of survival time was determined by the log-rank test. $\mathrm{P}<0.05$ was considered to indicate a statistically significant difference.

\section{Results}

Identification of DEGs in youth colorectal cancer. The gene expression levels of genes were downloaded from the GEO database. Based on GEO2R analysis, 6,322 and 12,585 DEGs in GSE41657 and GSE41258 were screened in youth colorectal cancer compared with normal colorectal tissues. Screened with the cut-off criteria of adjusted $\mathrm{P}<0.05$ and $\mid \log _{2} \mathrm{FCl}>1$, 1,256 upregulated and 1,782 downregulated genes were identified in the GSE41657 dataset (Fig. S1A), and 311 upregulated and 568 downregulated genes were identified in the GSE41258 

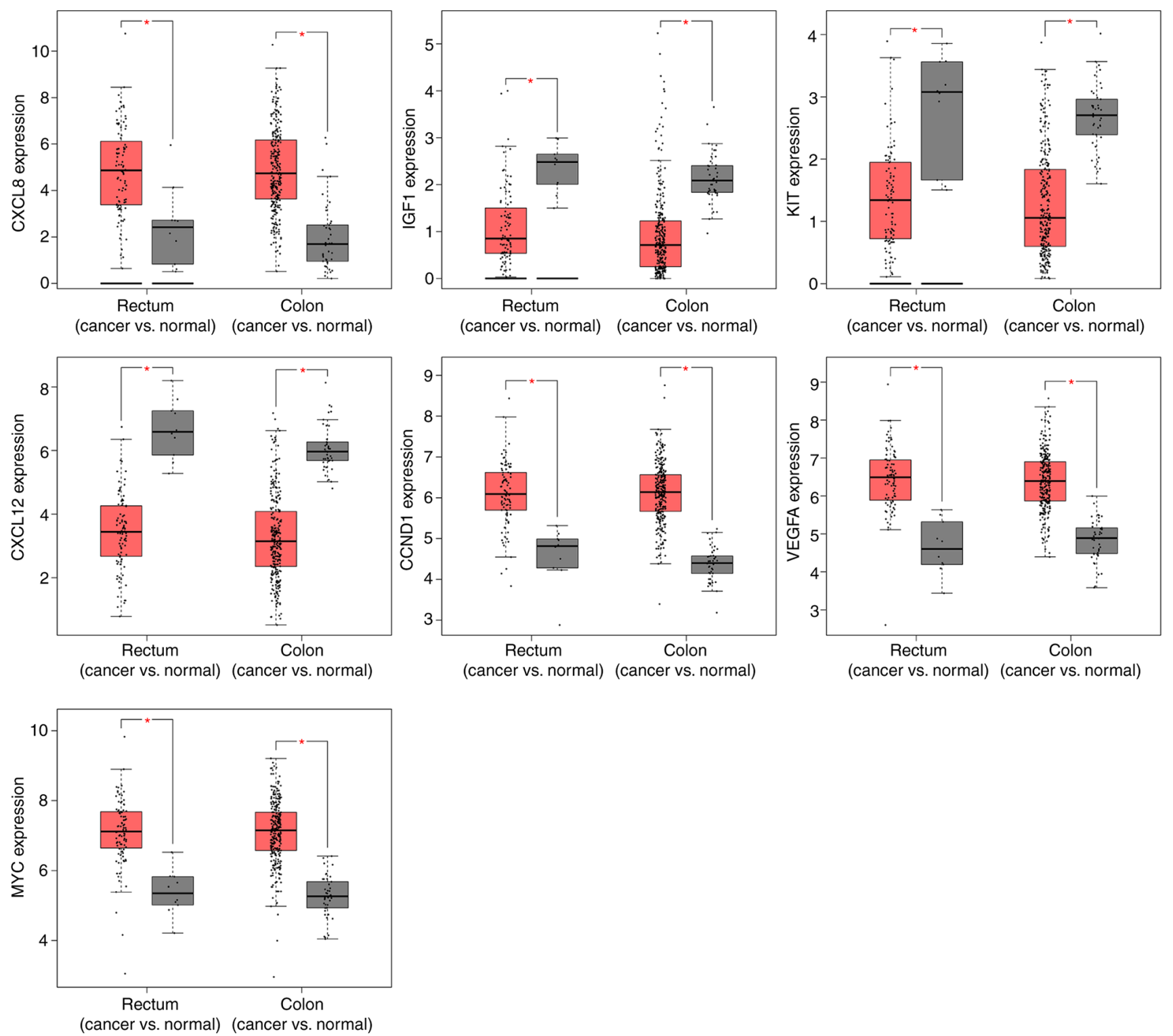

Figure 1. Differential expression of 7 candidate genes between colorectal cancer and normal tissues in The Cancer Genome Atlas database. Red represents cancer and gray normal tissues. $\mathrm{P}<0.05$.

dataset (Fig. S1B). In the integrated analysis of the two datasets, 443 overlapping genes, including 131 upregulated and 312 downregulated, were obtained (Fig. S1C and D).

$G O$ analysis of selected genes. The functional enrichment of 443 candidate genes was analyzed using DAVID. Three GO category results are presented in Table SII, including biological processes, cellular components and molecular functions. The biological process results revealed that the selected genes were mainly enriched in 'cellular response to zinc ion', 'negative regulation of growth' and 'cell proliferation'. The cellular component results revealed that selected genes were mainly enriched in 'extracellular exosome', 'extracellular space' and 'apical plasma membrane'. The molecular function analysis revealed that the selected genes were mainly enriched in 'protein binding', 'carbonate dehydratase activity' and 'CXCR chemokine receptor binding'. These results demonstrated that the majority of the candidate genes were significantly enriched in 'binding' and 'cell proliferation'.
Signaling pathway enrichment analysis. The signaling pathway enrichment of 443 candidate genes was analyzed using KEGG pathway online databases. A total of 23 genes were significantly enriched in 'Pathways in cancer' (Table SIII). Based on previous reports $(15,16)$, 'Pathways in cancer' is an important signaling pathway associated with the occurrence and development of cancer.

Protein-protein network construction. A total of 443 candidate genes were analyzed using the STRING 11.0 database, and then the protein-protein interaction (PPI) was imported into Cytoscape 3.6.0 software to build a visible network diagram. A total of 390 nodes and 1,462 edges are presented in the network (Fig. S2).

Hub gene selection and analysis with key signaling pathway. Within the PPI network, the cytoHubba plugin was used to screen for hub genes. Based on the degree algorithm, the top 10 hub genes were VEGFA, CXCL8, MYC, CD44, CXCL12, 

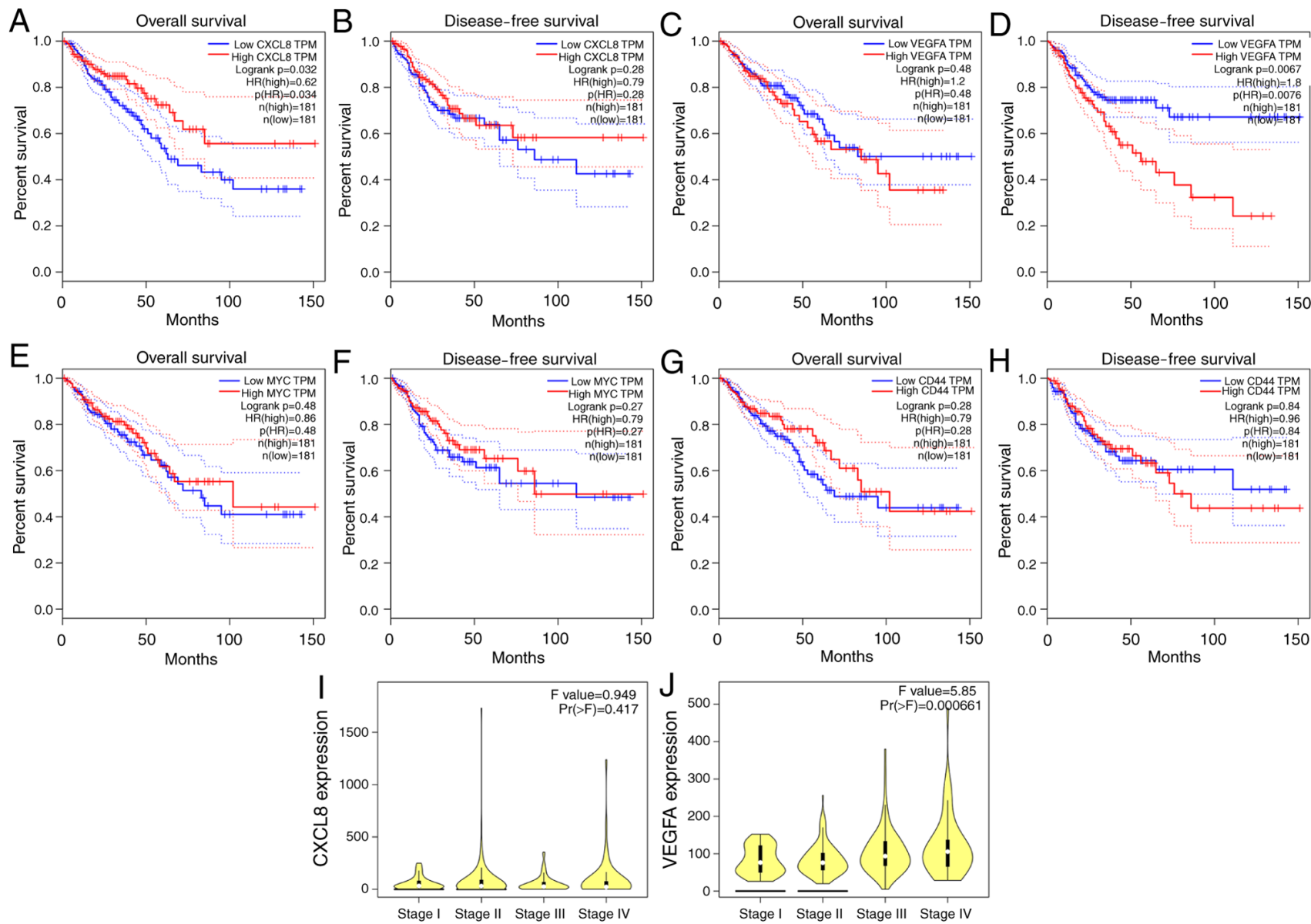

Figure 2. Association of gene expression level with survival and tumor staging. Association of the expression levels of (A and B) CXCL8, (C and D) VEGFA, (E and F) MYC, and (G and H) CD44 with overall survival or disease-free survival. Association of (I) CXCL8 (P>0.05) and (J) VEGFA (P<0.05) expression level with tumor staging. CXCL8, interleukin-8.

CCND1, IGF1, CXCL1, KIT and SOX9 (Table I). Combining signaling pathway analysis, CXCL8, IGF1, KIT, CXCL12, CCND1, VEGFA and MYC in the ten hub genes were enriched in the 'pathways in cancer' (Fig. S3). Among these genes, CXCL8, CCND1, VEGFA and MYC were upregulated, and the others downregulated.

TCGA, HPA validation and survival analysis. Differential expression of CXCL8, IGF1, KIT, CXCL12, CCND1, VEGFA and MYC in colorectal cancer compared with normal colorectal tissues, was screened in TCGA database. The expression levels of these eight genes were significantly higher in both colon and rectal cancer tissues, as compared with normal colorectal tissues $(\mathrm{P}<0.05$; Fig. 1$)$.

These genes were further subjected to survival analysis to screen out genes associated with colorectal cancer prognosis. The expression level of CXCL8 was found to impact overall survival (OS; $\mathrm{P}<0.05$; Fig. $2 \mathrm{~A}$ ), and that of VEGFA to impact disease-free survival (DFS; $\mathrm{P}<0.05$; Fig. 2D). The expression level of MYC and CD44 was found to have no impact on OS or DFS (P>0.05; Fig. 2E-H). Thus, CXCL8 and VEGFA were identified as key genes.

Compared with the expression at different Tumor-NodeMetastasis (TNM) stages of colorectal cancer, it was found that the expression of the CXCL8 gene in high TNM-stage colorectal cancer was increased, but there was no statistical significance ( $\mathrm{P}>0.05$; Fig. 2I), while the gene expression of VEGFA in high TNM stages was significantly higher $(\mathrm{P}=0.000661$; Fig. $2 \mathrm{~J})$.

The comparison of CXCL8 and VEGFA expression between youth and elderly colorectal cancer, revealed no significant differences (Fig. 3A and B). Further analysis indicated that high CXCL8 and VEGFA expression was associated with tumor stage. The proportion of stages III-IV was $56.25 \%$ in youth colorectal cancer, $42.95 \%$ in elderly colorectal cancer with high CXCL8 expression, and $65.63 \%$ in youth colorectal cancer, $47.65 \%$ in elderly colorectal cancer with high VEGFA expression (Fig. 3F). The HPA database was also used to verify the protein expression levels of key genes. The expression of CXCL8 and VEGFA in colorectal cancer from the HPA database was also consistent with the aforementioned bioinformatics analysis (Fig. 4C and D).

$R T$-qPCR validation. To confirm the reliability of the findings from data mining, the two genes, CXCL8 and VEGFA, were selected for validation by RT-qPCR in six pairs of youth colorectal cancer and adjacent normal tissues. According to the experimental results, the difference in expression of these two genes is consistent with the results of data mining. In youth colorectal cancer, the expression level of CXCL8 and VEGFA were significantly upregulated $(\mathrm{P}<0.05$; Fig. 4A and B). 

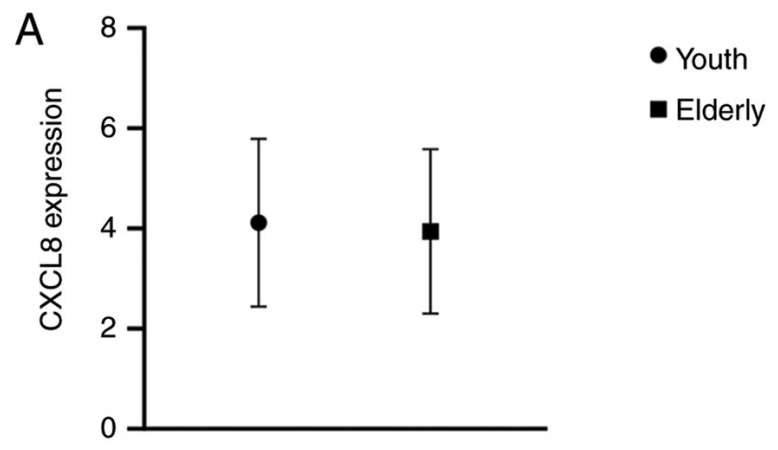

C
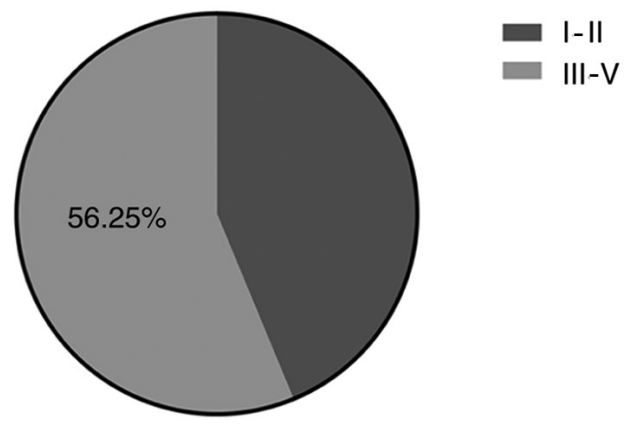

Stage in youth with high CXCL8
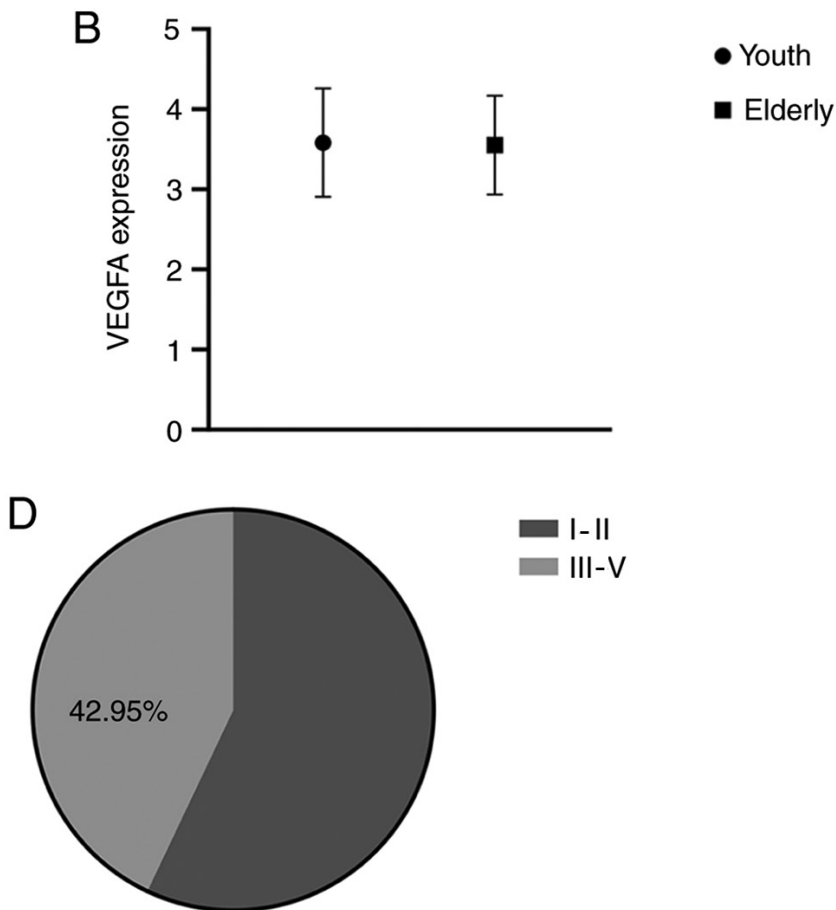

Stage in elderly with high CXCL8

$E$

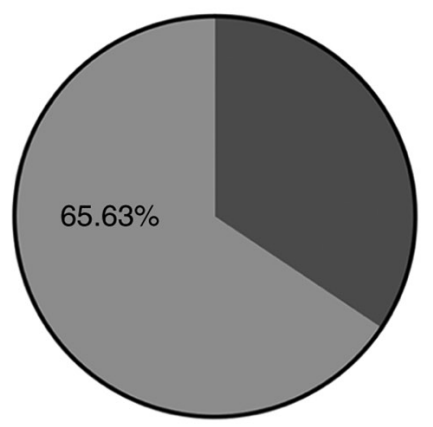

Stage in youth with high VEGFA

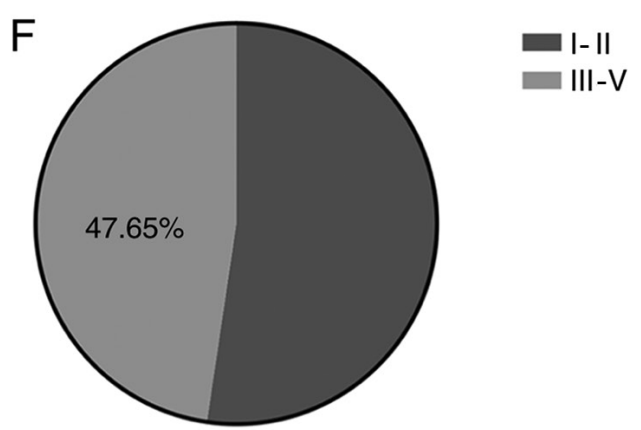

Stage in elderly with high VEGFA

Figure 3. (A and B) No significant differences in key genes expression were found between youth colorectal cancer and elderly colorectal cancer. (C-F) Among patients with high key genes expression, higher proportion of stage III-IV patients was found in youth colorectal cancer compared with elderly colorectal cancer.

\section{Discussion}

Colorectal cancer is a common malignant tumor of the digestive system, which seriously threatens human health. According to the 2018 Global Cancer Data Statistics Report, among malignant tumors, colorectal cancer ranks third in morbidity and second in mortality (17). In recent years, the incidence and mortality of colorectal cancer have been on the rise, particularly in young people. The onset of colorectal cancer in young people is typically undetected, and most patients are diagnosed during the middle and late stages of the disease, at which point, the best treatment opportunities are no longer suitable (18). Therefore, early diagnosis of young patients with colorectal cancer to ensure early treatment is particularly important for improving survival time and prognosis. With the advent of precision medicine, it is necessary to explore biological markers that are closely associated with the diagnosis, prognosis and treatment of colorectal cancer in young people.
In the present study, through bioinformatics data mining combined with gene annotation and signaling pathway analysis, two genes closely associated with young colorectal cancer, CXCL8 and VEGFA, were screened out. The difference in the expression of these two genes was confirmed by RT-qPCR. According to the data from TCGA database, the expression of these two genes was closely associated with the survival time of patients with colorectal cancer.

Both CXCL8 and VEGFA are cytokines that promote angiogenesis, and play an important role in tumor growth and metastasis (19). CXCL8, also known as interleukin 8, is a member of the chemokine family. CXCL8 is mainly produced by macrophages, and endothelial, epithelial and other cells (20). The sequence encoding CXCL8 is located on chromosome 4q13-21 (21). CXCL8 has been found to be highly expressed in various types of cancer, such as non-small cell lung (22), gastric (23), breast (24) and colorectal cancer (25). Studies have reported that, in colorectal cancer, CXCL8 mainly induces epithelial-mesenchymal transition through 
A

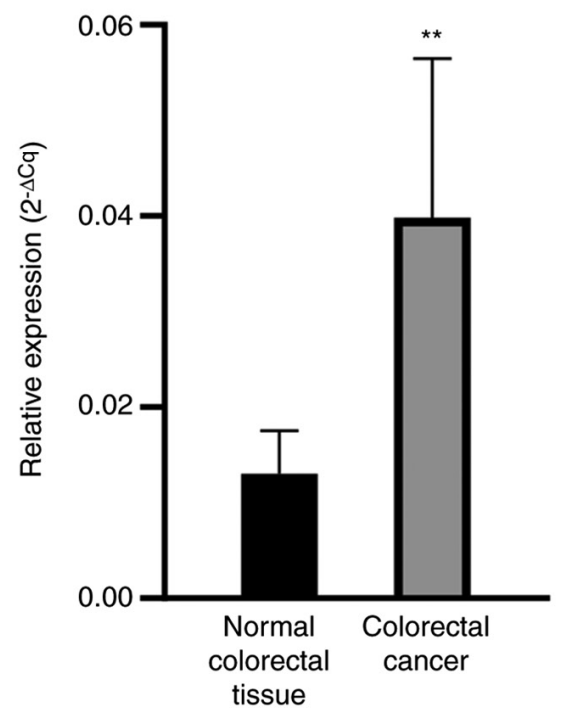

C

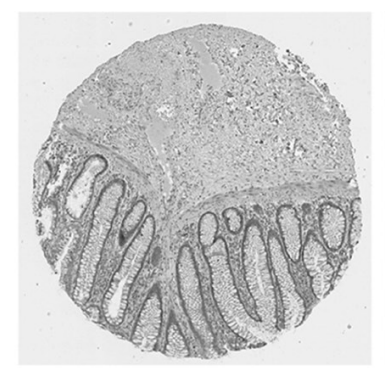

Normal colorectal tissue
CXCL8

CXCL8

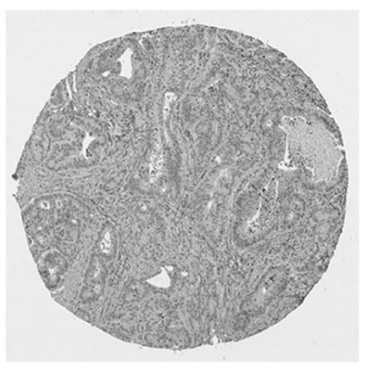

Colorectal cancer
B

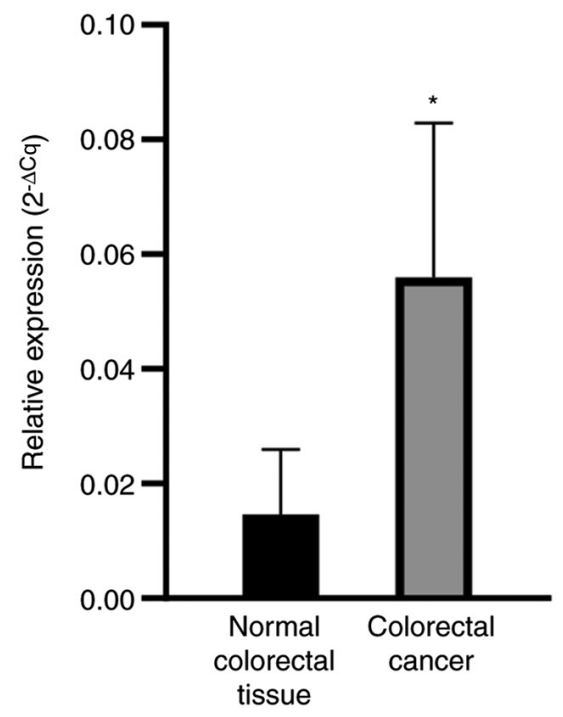

D

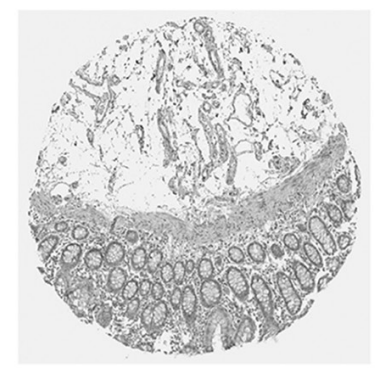

Normal colorectal tissue
VEGFA

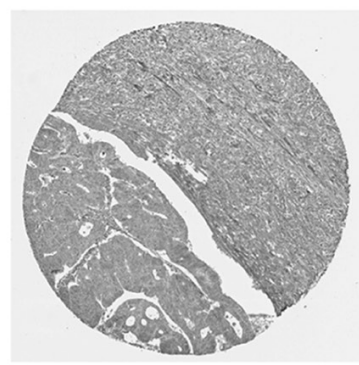

Colorectal cancer

Figure 4. Validation of key genes expression at the transcriptional and translational level by reverse transcription-quantitative PCR and the Human Protein Atlas database. (A and B) CXCL8 and VEGFA expression in colorectal cancer and normal colorectal tissues. (C and D) Protein expression of CXCL8 and VEGFA in colorectal cancer and normal colorectal tissues based on the Human Protein database. Magnification, $\mathrm{x} 100$. ${ }^{*} \mathrm{P}<0.05$ and ${ }^{* *} \mathrm{P}<0.01$. CXCL8, interleukin-8.

the PI3K/Akt/NF-кB signaling pathway (25). Certain studies have reported that the low expression of CXCL8 is associated with poor prognosis of patients with colorectal cancer $(26,27)$. This view is consistent with the results of the present study. As CXCL8 is a cancer-promoting factor. The reason for the association between its high expression and favorable prognosis seems to be contradictory, and the underlying mechanism remains unclear. This favorable prognosis may be associated with other functions of CXCL8. For example, CXCL8 as a chemokine has the ability to guide neutrophils to infectious diseases and control infection (28).

VEGFA is a member of the vascular endothelial growth factor (VEGF) protein family (29). VEGFA is the main regulator of angiogenesis, and its combination with VEGFR2 promotes the proliferation of endothelial cells through the RAS-RAF-MAPK-ERK signaling pathway (30). VEGFA is widely distributed in the body, and the high expression of VEGFA can be seen in a variety of cancer types, such as lung (31), gastric (32), breast (33), ovarian (34) and colorectal cancer (35). VEGFA can promote angiogenesis, thus it is useful to understand that the high expression of VEGFA promotes tumor growth, which in turn leads to a worse prognosis. In the present study, it was found that compared with the high VEGFA expression, the low VEGFA expression prolongs the survival time of patients with colorectal cancer, particularly the DFS. Moreover, colorectal cancer with a high VEGFA expression is associated with a higher disease stage. In recent years, various therapeutic targets for VEGFA have been manufactured. Bevacizumab was the first VEGFA inhibitor approved by the US Food and Drug Administration in 2004 for the treatment of metastatic colorectal cancer; since then, this drug has been widely used in clinical treatment and is known to significantly prolong the survival time of multiple patients (36).

The present study was not, however, without its limitations. Firstly, the number of specimens used to verify the results of the study was small. Secondly, no prospective experiments have been conducted to verify the association between candidate genes and patient prognosis.

In conclusion, through GEO datasets, TCGA database analysis and RT-qPCR validation, two key genes associated with prognosis, CXCL8 and VEGFA, were identified in youth colorectal cancer. These key genes were enriched in the 'pathways in cancer', and may be ideal prognostic indicators or therapeutic targets for youth colorectal cancer. 


\section{Acknowledgements}

Not applicable.

\section{Funding}

The present study was supported by The Open Foundation of Engineering Research Center of Big Data Application in Private Health Medicine, The Fujian Province University (grant no. KF2020009) and The National Natural Science Foundation of China (grant no. 81860334).

\section{Availability of data and materials}

The datasets used during the present study are available from the corresponding author on reasonable request.

\section{Authors' contributions}

JC performed the experiments and was a major contributor in writing the manuscript. ML and YC downloaded the datasets and performed statistical analysis. JC and JG analyzed the obtained data. WL designed the study. All authors have read and approved the final manuscript.

\section{Ethics approval and consent to participate}

The present study was approved by the Ethics Committee of The Affiliated Hospital of Putian University (approval no. 202016). Written informed consent was obtained from each patient for sample collection and analysis.

\section{Patient consent for publication}

Not applicable.

\section{Competing interests}

The authors declare that they have no competing interests.

\section{References}

1. Naeem A, Tun AM and Guevara E: Molecular genetics and the role of molecularly targeted agents in metastatic colorectal carcinoma. J Gastrointest Cancer 51: 387-400, 2020.

2. Weinberg BA, Marshall JL and Salem ME: The growing challenge of young adults with colorectal cancer. Oncology (Williston Park) 31: 381-389, 2017

3. Arnold M, Sierra MS, Laversanne M, Soerjomataram I, Jemal A and Bray F: Global patterns and trends in colorectal cancer incidence and mortality. Gut 66: 683-691, 2017.

4. Ravegnini G and Angelini S: Toward precision medicine: How far is the goal? Int J Mol Sci 17: 245, 2016.

5. Johnson TM: Perspective on precision medicine in oncology. Pharmacotherapy 37: 988-989, 2017.

6. Wei Q: Personalized medicine at a prime time for cancer medicine-introducing cancer medicine. Cancer Med 1: 1, 2012.

7. Mork ME, You YN, Ying J, Bannon SA, Lynch PM, Rodriguez-Bigas MA and Vilar E: High prevalence of hereditary cancer syndromes in adolescents and young adults with colorectal cancer. J Clin Oncol 33: 3544-3549, 2015.

8. Khan SA, Morris M, Idrees K, Gimbel MI, Rosenberg S, Zeng Z, Li F, Gan G, Shia J, LaQuaglia MP and Paty PB: Colorectal cancer in the very young: A comparative study of tumor markers, pathology and survival in early onset and adult onset patients. J Pediatr Surg 51: 1812-1817, 2016.
9. Magnani G, Furlan D, Sahnane N, Reggiani Bonetti L, Domati F and Pedroni M: Molecular features and methylation status in early onset ( $\leq 40$ years) colorectal cancer: A population based, case-control study. Gastroenterol Res Pract 2015: 132190, 2015.

10. Pearlman R, Frankel WL, Swanson B, Zhao W, Yilmaz A Miller K, Bacher J, Bigley C, Nelsen L, Goodfellow PJ, et al: Prevalence and spectrum of germline cancer susceptibility gene mutations among patients with early-onset colorectal cancer. JAMA Oncol 3: 464-471, 2017.

11. Barrett T, Wilhite SE, Ledoux P, Evangelista C, Kim IF, Tomashevsky M, Marshall KA, Phillippy KH, Sherman PM, Holko M, et al: NCBI GEO: Archive for functional genomics data sets-update. Nucleic Acids Res 41 (Database Issue): D991-D995, 2013.

12. Tang Z, Li C, Kang B, Gao G, Li C and Zhang Z: GEPIA: A web server for cancer and normal gene expression profiling and interactive analyses. Nucleic Acids Res 45 (W1): W98-W102, 2017.

13. Uhlén M, Fagerberg L, Hallström BM, Lindskog C, Oksvold P, Mardinoglu A, Sivertsson Å, Kampf C, Sjöstedt E, Asplund A, et al: Proteomics. Tissue-based map of the human proteome. Science 347: 1260419, 2015.

14. Schmittgen TD and Livak KJ: Analyzing real-time PCR data by the comparative C(T) method. Nat Protoc 3: 1101-1108, 2008

15. Yang L, Wang J, Sun X, Cao Y, Ning S, Zhang H, Chen L, Li R, Tian Q, Wang L, et al: Identifying a polymorphic 'switch' that influences miRNAs' regulation of a myasthenia gravis risk pathway. PLoS One 9: e104827, 2014.

16. Zhou X, Zheng R, Zhang $\mathrm{H}$ and He T: Pathway crosstalk analysis of microarray gene expression profile in human hepatocellular carcinoma. Pathol oncol Res 21: 563-569, 2015.

17. Bray F, Ferlay J, Soerjomataram I, Siegel RL, Torre LA and Jemal A: Global cancer statistics 2018: GLOBOCAN estimates of incidence and mortality worldwide for 36 cancers in 185 countries. CA Cancer J Clin 68: 394-424, 2018.

18. Chen W, Zheng R, Baade PD, Zhang S, Zeng H, Bray F, Jemal A, $\mathrm{Yu}$ XQ and He J: Cancer statistics in China, 2015. CA Cancer J Clin 66: 115-132, 2016.

19. Marjon PL, Bobrovnikova-Marjon EV and Abcouwer SF Expression of the pro-angiogenic factors vascular endothelial growth factor and interleukin-8/CXCL8 by human breast carcinomas is responsive to nutrient deprivation and endoplasmic reticulum stress. Mol Cancer 3: 4, 2004.

20. Matsushima K, Baldwin ET and Mukaida N: Interleukin-8 and MCAF: Novel leukocyte recruitment and activating cytokines. Chem Immunol 51: 236-265, 1992.

21. Liu Q, Li A, Tian Y, Wu JD, Liu Y, Li T, Chen Y, Han X and Wu K: The CXCL8-CXCR1/2 pathways in cancer. Cytokine Growth Factor Rev 31: 61-71, 2016.

22. Masuya D, Huang C, Liu D, Kameyama K, Hayashi E, Yamauchi A, Kobayashi S, Haba R and Yokomise H: The intratumoral expression of vascular endothelial growth factor and interleukin- 8 associated with angiogenesis in nonsmall cell lung carcinoma patients. Cancer 92: 2628-2638, 2001

23. Yamamoto Y, Kuroda K, Sera T, Sugimoto A, Kushiyama S, Nishimura S, Togano S, Okuno T, Yoshii M, Tamura T, et al: The clinicopathological significance of the CXCR2 ligands, CXCL1, CXCL2, CXCL3, CXCL5, CXCL6, CXCL7, and CXCL8 in gastric cancer. Anticancer Res 39: 6645-6652, 2019.

24. Liubomirski Y, Lerrer S, Meshel T, Morein D, RubinsteinAchiasaf L, Sprinzak D, Wiemann S, Körner C, Ehrlich M and Ben-Baruch A: Notch-mediated tumor-stroma-inflammation networks promote invasive properties and CXCL8 expression in triple-negative breast cancer. Front Immunol 10: 804, 2019.

25. Shen T, Yang Z, Cheng X, Xiao Y, Yu K, Cai X, Xia C and Li Y: CXCL8 induces epithelial-mesenchymal transition in colon cancer cells via the PI3K/Akt/NF- $\kappa \mathrm{B}$ signaling pathway. Oncol Rep 37: 2095-2100, 2017.

26. Doll D, Keller L, Maak M, Boulesteix AL, Siewert JR, Holzmann B and Janssen KP: Differential expression of the chemokines GRO-2, GRO-3, and interleukin-8 in colon cancer and their impact on metastatic disease and survival. Int $\mathrm{J}$ Colorectal Dis 25: 573-581, 2010.

27. Zhao QQ, Jiang C, Gao Q, Zhang YY, Wang G, Chen XP, Wu SB and Tang J: Gene expression and methylation profiles identified CXCL3 and CXCL8 as key genes for diagnosis and prognosis of colon adenocarcinoma. J Cell Physiol 235: 4902-4912, 2020.

28. Das ST, Rajagopalan L, Guerrero-Plata A, Sai J, Richmond A, Garofalo RP and Rajarathnam K: Monomeric and dimeric CXCL8 are both essential for in vivo neutrophil recruitment. PLoS One 5: e11754, 2010. 
29. Ferrara N: VEGF and the quest for tumour angiogenesis factors. Nat Rev Cancer 2: 795-803, 2002.

30. Herbert SP and Stainier DY: Molecular control of endothelial cell behaviour during blood vessel morphogenesis. Nat Rev Mol Cell Biol 12: 551-564, 2011.

31. Zhang SD, McCrudden CM and Kwok HF: Prognostic significance of combining VEGFA, FLT1 and KDR mRNA expression in lung cancer. Oncol Lett 10: 1893-1901, 2015.

32. Lu J, Wang YH, Yoon C, Huang XY, Xu Y, Xie JW, Wang JB, Lin JX, Chen QY, Cao LL, et al: Circular RNA circ-RanGAP1 regulates VEGFA expression by targeting miR-877-3p to facilitate gastric cancer invasion and metastasis. Cancer Lett 471 : $38-48,2020$.

33. Korobeinikova E, Ugenskiene R, Insodaite R, Rudzianskas V, Jaselske E, Poskiene L and Juozaityte E: Association of angiogenesis and inflammation-related gene functional polymorphisms with early-stage breast cancer prognosis. Oncol Lett 19: 3687-3700, 2020.
34. Zhou P, Xiong T, Chen J,Li F, Qi T and Yuan J: Clinical significance of melanoma cell adhesion molecule CD146 and VEGFA expression in epithelial ovarian cancer. Oncol Lett 17: 2418-2424, 2019.

35. Wang R, Ma Y, Zhan S, Zhang G, Cao L, Zhang X, Shi T and Chen W: B7-H3 promotes colorectal cancer angiogenesis through activating the NF- $\kappa \mathrm{B}$ pathway to induce VEGFA expression. Cell Death Dis 11: 55, 2020.

36. Ferrara $\mathrm{N}$ and Adamis AP: Ten years of anti-vascular endothelial growth factor therapy. Nat Rev Drug Discov 15: 385-403, 2016.

This work is licensed under a Creative Commons Attribution-NonCommercial-NoDerivatives 4.0 International (CC BY-NC-ND 4.0) License. 